\title{
Recognition of Infected Erythrocytes by Inclusion Tree Representation and Parasitemia Estimation in Blood Smear Images \\ Doke Pranoti R.; Doke Pooja R
}

*Lecturer, Government Residence Womens Polytechnic, Tasgaon, Maharashtra, India

\begin{abstract} in early stage.

1. Preprocessing, Histogram and Segmentation

2. Inclusion-Tree representation

3. Splitting of clumped erythrocytes

4. Counting and labeling

5. Cell stage identification

6. Feature extraction and classification
\end{abstract}

Blood cells are composed of erythrocytes (red blood cells, RBCs), leukocytes (white blood cells, WBCs) and thrombocytes (platelets). Both WBC and RBC have fixed count in our body. If their count is less than the ideal count then it is an indication that our body is not healthy. Hence blood count helps in detecting many diseases

According to World Health Organization about 3.2 billion people are at risk of malaria[2]. But, malaria is preventable and curable, if the patient is correctly diagnosed in early stage.

The proposed approach to diagnose malaria mainly consists of following steps:

The algorithm is used to count malaria infected RBC in blood smear. A clump splitting method is used for precise RBC counting. Cell stage identification is performed in this approach by calculating Equivalent Circular Diameter. Quantification method improves overall performance in the determination of stages of infection such as ring, trophozoite and Schizont. Percentage of Parasitemia is calculate.

Keywords : Segmentation, Clumped Cells, Plasmodium Falciparum, Inclusion Tree Representation, Counting, Labeling

\section{INTRODUCTION}

Blood is a connective tissue consisting of cells suspended in plasma. Blood transport various agents such as oxygen, carbon dioxide, nutrients, wastes, and hormones. Blood cells are composed of erythrocytes, leukocytes and thrombocytes. Both WBC and RBC have fixed count in our body. If their count is less than the ideal count then it is an indication that our body is not healthy. Malaria, caused by a protozoan parasites of the genus Plasmodium, transmitted to people through the bites of infected mosquitoes, is the most serious and widespread parasitic disease of humans. Plasmodium falciparum is the most virulent than other species.

But, malaria is preventable and curable, if the patient is correctly diagnosed at early stage.

Recent progress based on microscopic imaging has given significant contribution in diagnosis of malaria 
infection based on blood images. Due to the requirement of prompt and precise diagnosis of malaria, the current study has proposed a color image Clump splitting and segmentation of malaria parasites using Inclusion tree representation. The proposed clump splitting method provides a basic step for detection of the presence of malaria parasites and counting of infected cells in blood smears. In this approach all cells are preprocessed by digital image processing techniques. Overlapping cells and Parasites are segmented from infected erythrocytes by inclusion tree representation method.

After this, Cells infected with malaria parasite are counted. In this approach we can conclude the stage of parasite. Individual cells are identified by pixels are connected to each other. Each group of connected pixels will be given a label, a number, to identify it and distinguish it from the other cells. Each Cell is assigned with a different color to visually distinct cells. Parasite Infected Cell stages with Ring, Trophozoite and schizont are differentiated and labeled accordingly.

\section{LITERATURE SURVEY}

The morphological cell classification is done by experienced operators still it is not precise, accurate and it is time consuming. In traditional, blood cell analysis is done by complete blood cell count (CBC). So, now a days many image processing approaches are there for counting of infected cells and diagnosis of diseases.

Application of different Image segmentation algorithms have led to precise quantifications and diagnosis. Following techniques are used for this purpose.

Local threshold levels were used for enhancing the early life stage detection.
The parasites are detected by means of an automatic thresholding based on morphological approach, to evaluate size of RBCs and nuclei of parasite.

Template matching strategy is used for detection of erythrocytes. These different type of approaches were used.

\section{METHODOLOGY}

The proposed algorithm for Splitting of clumped erythrocytes, counting of infected cells and cell stage identification are summarized as follows:

Step 1: Capture stained blood smear images

Step 2 : Histogram plotting

Step 3: Perform morphological operations

Step 4: Inclusion tree representation approach is used for splitting of clumped erythrocytes.

Step 5: Segmentation of overlapping objects.

Step 6: Splitting of clumped erythrocytes and total number of cells are shown in figure by red color circles.

Step 7: Counts the no of cells irrespective of infection

Step 8: Each group of connected pixels will be given a label, a number. Calculate number of infected erythrocytes.

Step 9: Calculate Equivalent Circular Diameter (ECD).

Step10: Cell stages are identified on the basis of Equivalent circular diameter.

Step11: Calculate percentage of parasitemia.

A. Image Pre-processing

In this, after image acquisition microscopic images are processed with digital image processing techniques. Histogram of the image is calculated depending on threshold value image is processed further. 


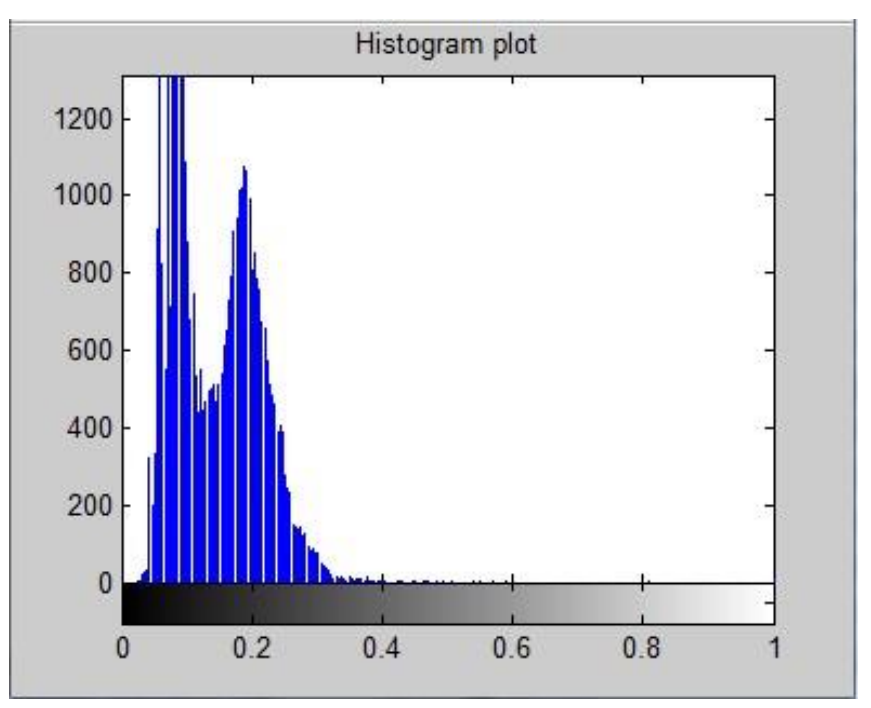

Fig 1: Histogram plot

Image processing morphological techniques such as image enhancement, erosion, filtering, holes filling, edge detection are performed[1]. The YCbCr transformation is performed on $\mathrm{m}^{*} \mathrm{n}$ RGB image to decorrelate it into luminance and chrominance components.

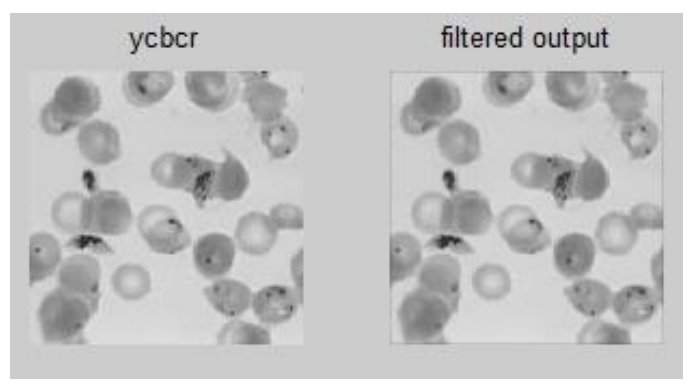

Fig. 2: (a)YCbCr color transformation (b) filtered output

B. Recognition of erythrocytes

Our aim is to recognize erythrocytes and count it. Here, pixels are labeled and erythrocytes are recognized as a foreground pixels.

a. Inclusion tree representation

This image processing algorithm is provides relation between foreground and background pixels. Total numbers of cells are counted. Morphological operations are performed to find region of interest.
Filtering is performed to remove artifacts [3]. It provides the hierarchical representation of enclosure relationship between contours.

Erythrocytes touching image boundaries are incomplete forms and are removed. Foreground objects with a largest area than a threshold are considered as clumped erythrocytes and these are separated for total quantification.

b. Color space classification and pixel labeling Erythrocytes infected with malaria parasite are distinguished by color space classification by separating background and foreground objects [3]. Cells are assigned with different color to visually show the user the distinct cells.

\section{Splitting of clumped erythrocytes}

The main problem for quantification of erythrocytes is presence connected cells and overlapping cells. In this approach clumped cells are separated by chain code representation of erythrocytes and connected cells [3],[5]. Total number of cells are counted irrespective of infection and plotted with dark circles. Individual cells are identified by pixels connected to each other.

Edges of a color image are calculated by the maxgradient method as follows[6],

$$
\begin{aligned}
& {\left[\begin{array}{l}
r x, g x, b x \\
r y, g y, b y
\end{array}\right] *\left[\begin{array}{l}
r x, r y \\
g x, g y
\end{array}\right]} \\
& =[b x, b y] *\left[\begin{array}{l}
r x^{\wedge} 2+g x^{\wedge} 2+b x^{\wedge} 2, r x^{*} r y+g x^{*} g y+b x^{*} b y \\
r x^{*} r y+g x^{*} g y+b x^{*} b y, r y^{\wedge} 2+g y^{\wedge} 2+b y^{\wedge} 2
\end{array}\right] \\
& =\left[\begin{array}{l}
J x, J x y \\
J x y, J y
\end{array}\right]
\end{aligned}
$$

$\mathrm{J}$ is the jacobian, its elements are the partial derivatives of $\mathrm{r} / \mathrm{g} / \mathrm{b}$ with respect to $\mathrm{x} / \mathrm{y}$. Eigen values are used as template. 


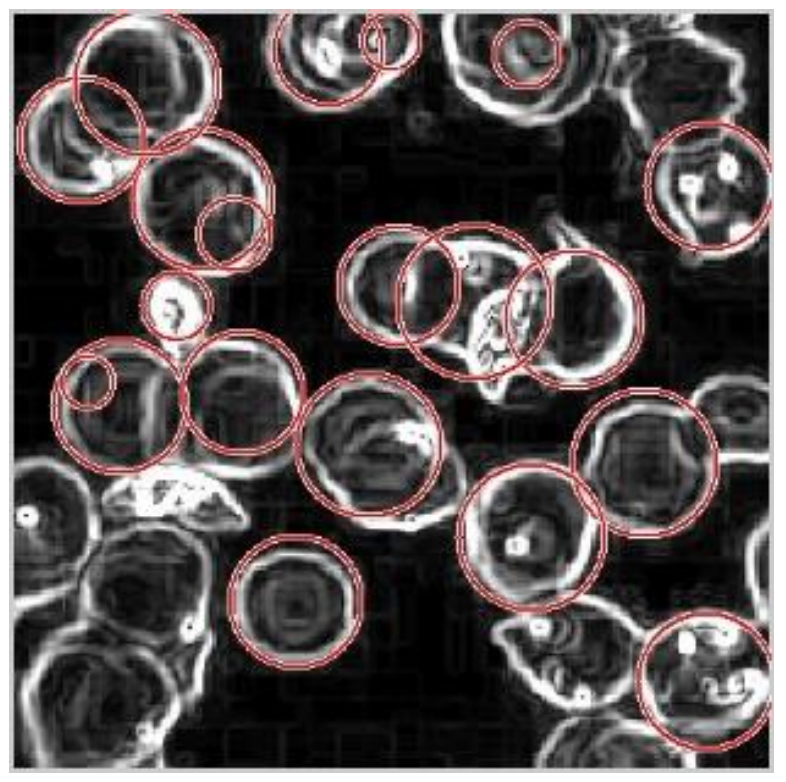

Fig 3: Total number of cells are shown by red color circles

D. Detection of erythrocytes infected with parasites Individual pixels connected to each other are identified and are labeled at its centers.

These parasites are shown by different colors. Connected component trees of lower (0) and upper (255) values are built. Infected erythrocyte holes are into the lower value tree for each of the connected components.

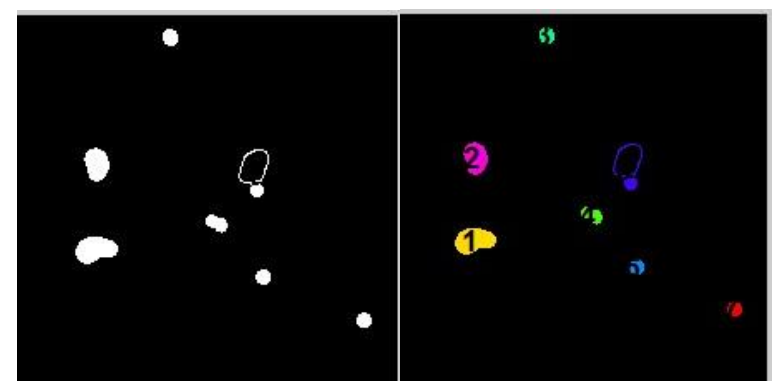

Fig.4: (a) Holes (b) labeled image of infected erythrocytes

E. Cell stage identification

This is most important step in identification of erythrocytes infected with malaria parasite because visually it is not possible to identify the size and other parameters of parasite and its transition from one stage to another. So, this cell stage by this cell stage identification we can distinguish ring, trophozoite and schizont stages. Based on Equivalent Circular Diameter (ECD)cell stages are indetified.

By knowing cell stage diagnosis of disease can be done and if it is at early stage then it can be cured. There are 3 stages considered in this paper that are ring stage, trophozoital stage and schizonts stage. Cells ECD can be calculated as,

$$
E C D=4 * \text { are a } \text { of } \mathrm{cell} / \pi
$$

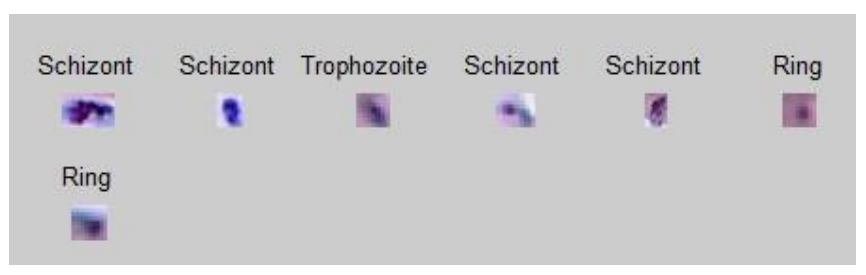

Fig. 5 : Cell stage identification such as ring, trophozoite and schizont stages is identified.

\section{F. Parasitemia Calculation}

It is calculated from total number of erythrocytes and infected erythrocytes[1].

Percentage of Parasitemia $=($ Infected Cells $/$ Total number of cells)*100

\section{G. Feature extraction and classification by SVM classifier}

In this paper, Features are extracted to classify image is infected with malaria or not.

Following are the features extracted from image, Contrast, correlation, energy and Homogeneity. Plot of energy and correlation can be plotted as,

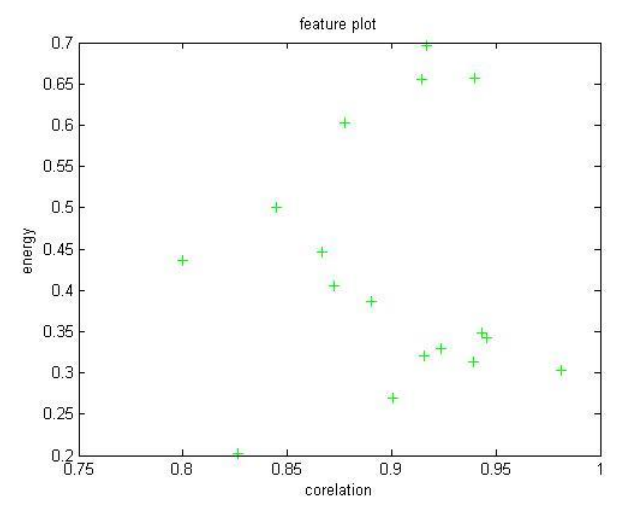

Fig 7 : Feature extraction plot 


\section{EXPERIMENTAL RESULTS}

In this paper, following results are obtained. Total number of erythrocytes are counted. Erythrocytes infected with malaria parasite are counted. Stages of infection such as ring stage, trophozoital stage and schizont stage can be identified[3].

Percentage of parasitemia is calculated. Following are the results in matlab command window:

\section{Total Cell count}

cel11 $=$

20

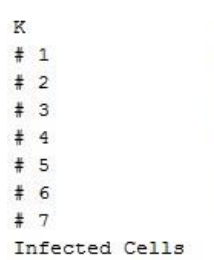

mean
81.4
80.9
37.6
14.7
21.0
20.8
43.3

intensity $288.0 \quad 66.3$ 215.0

15.0

84.0
92.0

109.0

65.0

65.0

$66.3 \begin{array}{ccc}\text { perimeter centriod ECD } \\ 67.3 & 138.9 & 19.1\end{array}$

$\begin{array}{lll}48.1 & 88.3 & 16.5\end{array}$

$\begin{array}{llll}31.3 & 91.5 & 13.5 & 10.3\end{array}$

$\begin{array}{llll}37.0 & 119.1 & 123.7 & 10.8\end{array}$

$\begin{array}{rrrr}143.6 & 141.5 & 95.7 & 11 .\end{array}$

$\begin{array}{llll}27.3 & 146.2 & 155.0 & 9.1\end{array}$

Infected Cells

para $=$

7

Percentage of parasitemia is 35.0

the image is infected

Fig 8: Experimental results at command window

\section{CONCLUSION}

From this method we calculated total number of cells, number of erythrocytes infected with parasites and stages of infection. From this method we get the percentage of parasitemia estimation.

\section{REFERENCES}

[1]. R. C. Gonzalez and R. E. Woods, Digital Image Processing. 3rd ed.,Prentice Hall, 2007.

[2]. World Malaria Report 2011, World Health Organization, Geneva, 2011.

[3]. "A semi-automatic method for quantification and classification of erythrocytesinfected with malaria parasites in microscopic images" in 2009 Elsevier Journal of Biomedical Informatics 42 (2009) 296-307

[4]. Pranati Rakshit, Kriti Bhowmik, "Detection of presence of Parasites in Human RBC in Case of diagnosing malaria using Image Processing “ in 2013, IEEE conference.

[5]. www.mathworks.in/help/images/ref/regionpro ps.html

[6]. https://in.mathworks.com/matlabcentral/fileexc hange/28114-fast-edges-of-a-color-image-actual--color--not-converting-to-grayscale/content/coloredges.m

\section{Cite this article as :}

Doke Pranoti R., Doke Pooja R, "Recognition of Infected Erythrocytes by Inclusion Tree Representation and Parasitemia Estimation in Blood Smear Images", International Journal of Scientific Research in Science and Technology (IJSRST), Online ISSN : 2395-602X, Print ISSN : 2395-6011, Volume 6 Issue 1, pp. 308-312, January-February 2019.

Available at doi :

https://doi.org/10.32628/IJSRST196149

Journal URL : http://ijsrst.com/IJSRST196149 